\title{
The Lyon Consensus: Does It Differ From the Previous Ones?
}

\author{
Matteo Ghisa, ${ }^{1}$ Brigida Barberio, ${ }^{1}$ Vincenzo Savarino, ${ }^{2}$ Elisa Marabotto, ${ }^{2}$ Mentore Ribolsi, ${ }^{3}$ Giorgia Bodini, ${ }^{2}$ Fabiana Zingone, \\ Marzio Frazzoni, ${ }^{4}$ and Edoardo Savarino ${ }^{1 *}$
}

${ }^{1}$ Division of Gastroenterology, Department of Surgery, Oncology and Gastroenterology, University of Padua, Padua, Italy; ${ }^{2}$ Division of Gastroenterology, Department of Internal Medicine, University of Genoa, Genoa, Italy; ${ }^{3}$ Unit of Gastroenterology, Campus Bio-Medico University, Rome, Italy; and ${ }^{4}$ Pathophysiology Unit, Baggiovara Hospital, Modena, Italy

Gastroesophageal reflux disease (GERD) is a complex disorder with heterogeneous symptoms and a multifaceted pathogenetic basis, which prevent a simple diagnostic algorithm or any categorical classification. Clinical history, questionnaires and response to proton pump inhibitor (PPI) therapy are insufficient tools to make a conclusive diagnosis of GERD and further investigations are frequently required. The Lyon Consensus goes beyond the previous classifications and defines endoscopic and functional parameters able to establish the presence of GERD. Evidences for reflux include high-grade erosive esophagitis, Barrett's esophagus, and peptic strictures at endoscopy as well as esophageal acid exposure time $>6 \%$ on $\mathrm{pH}$-metry or combined pH-impedance monitoring. Even if a normal endoscopy does not exclude GERD, its combination with distal acid exposure time $<4 \%$ on off-PPI pH-impedance monitoring provides sufficient evidence refuting this diagnosis. Reflux-symptom association on pH-monitoring provides supportive evidence for reflux-triggered symptoms and may predict a better treatment outcome, when present. Also recommendations to perform pH-impedance "on" or "off" PPI are well depicted. When endoscopy and pH-metry or combined pH-impedance monitoring are inconclusive, adjunctive evidence from biopsy findings (eg, microscopic esophagitis), high-resolution manometry (ie, ineffective esophagogastric barrier and esophageal body hypomotility), and novel impedance metrics, such as mean nocturnal baseline impedance and post-reflux swallow-induced peristaltic wave index, can contribute to better identify patients with GERD. Definition of individual patient phenotype, based on the level of refluxate exposure, mechanism of reflux, efficacy of clearance, underlying anatomy of the esophagogastric junction, and clinical presentation, will lead to manage GERD patients with a tailored approach chosen among different types of therapy.

(J Neurogastroenterol Motil 2020;26:311-321)

\section{Key Words}

Endoscopy; Esophagitis; Gastroesophageal reflux; Manometry; Proton pump inhibitors

\section{Introduction}

Gastroesophageal reflux disease (GERD) is a condition which develops when the reflux of the stomach contents causes troublesome symptoms and/or complications. ${ }^{1}$ It involves up to $25 \%$ of the population in Western countries, with the highest rates reported in Europe and United States. ${ }^{2}$ Starting from the Genval Workshop (1999), through Porto Consensus (2004) and Montreal definition of GERD (2006), gastroenterologists tried to clarify this vague and faded topic, develop a clear definition of it, classify its manifestations in esophageal and extra-esophageal (EE) syndromes, and propose

Received: March 11, 2020 Revised: None Accepted: April 21, 2020

(c) This is an Open Access article distributed under the terms of the Creative Commons Attribution Non-Commercial License (http://creativecommons. org/licenses/by-nc/4.0) which permits unrestricted non-commercial use, distribution, and reproduction in any medium, provided the original work is properly cited.

*Correspondence: Edoardo Savarino, MD, PhD Department of Surgery, Oncology and Gastroenterology, University of Padua, Via Giustiniani, 2, 35128 Padova, Italy Tel: +39-049-8217749, Fax: +39-049-8760820, E-mail: edoardo.savarino@unipd.it 
more definite pathophysiological mechanisms. ${ }^{1,3,4}$

If it is true that GERD was initially a clinical diagnosis based on typical symptoms, there is no doubt that endoscopic findings, proton pump inhibitor (PPI) trial and questionnaire scores have helped to achieve this diagnosis. ${ }^{5}$ However, the label "GERD" started to be associated with a long list of signs and symptoms, sometimes without proven evidences, resulting in frequent misdiagnosis and misuse of medical therapies. ${ }^{6,7}$ Therefore, it has become more and more important to diagnose or to exclude GERD with enough confidence. A series of questionnaires, devices, and methods have been proposed to achieve this aim. The achievement of better endoscopic classifications and histological alterations as well as the widespread popularity of ambulatory reflux monitoring methods and esophageal high-resolution manometry (HRM) have rendered the diagnostic process more accurate, but even much more complex (Fig. 1).

The Lyon Consensus provides clear and practical information to obtain GERD diagnosis and to understand the wide spectrum of subgroups presenting similar symptoms. ${ }^{8}$ Thanks to this improved knowledge and phenotyping classification, a tailored therapy going beyond symptoms can now be offered to patients. The aims of this review are to point out progresses attained with Lyon Consensus compared with the previous ones, to summarize the recommendations therein contained, and to provide a perspective on future research needed in this area.

\section{First Approach to Gastroesophageal Reflux Disease Patients: Clinical History, Question- naires, and Proton Pump Inhibitor Trial}

Patient evaluation usually starts with the assessment of symptoms suspected for GERD (Fig. 2). A clinical history of typical symptoms, such as heartburn and regurgitation, is usually suggestive for GERD and predicts a good response to anti-secretive therapy. However, the above typical symptoms, even if collected by an expert gastroenterologist, reach at most about $70 \%$ sensitivity and specificity, compared to $\mathrm{pH}$-metry or endoscopy. ${ }^{9}$ As to EE symptoms, the diagnostic accuracy is much lower for both the welldefined gastroesophageal reflux (GER)-related syndromes (eg, cough and laryngitis) and the proposed ones, such as idiopathic pulmonary fibrosis. ${ }^{10,11}$

Also, questionnaires present the same limitations of clinical history in terms of poor diagnostic accuracy. Among the various questionnaires available, the gastroesophageal reflux disease questionnaire and the reflux disease questionnaire have been validated in multiple languages and have been shown to provide some diagnostic help. ${ }^{9,12,13}$ However, they have demonstrated only modest accuracy (less than 70\%) and, therefore, add little to a careful and precise outpatient clinical evaluation. ${ }^{9}$

In patients without red flags (eg, age, family history, weight

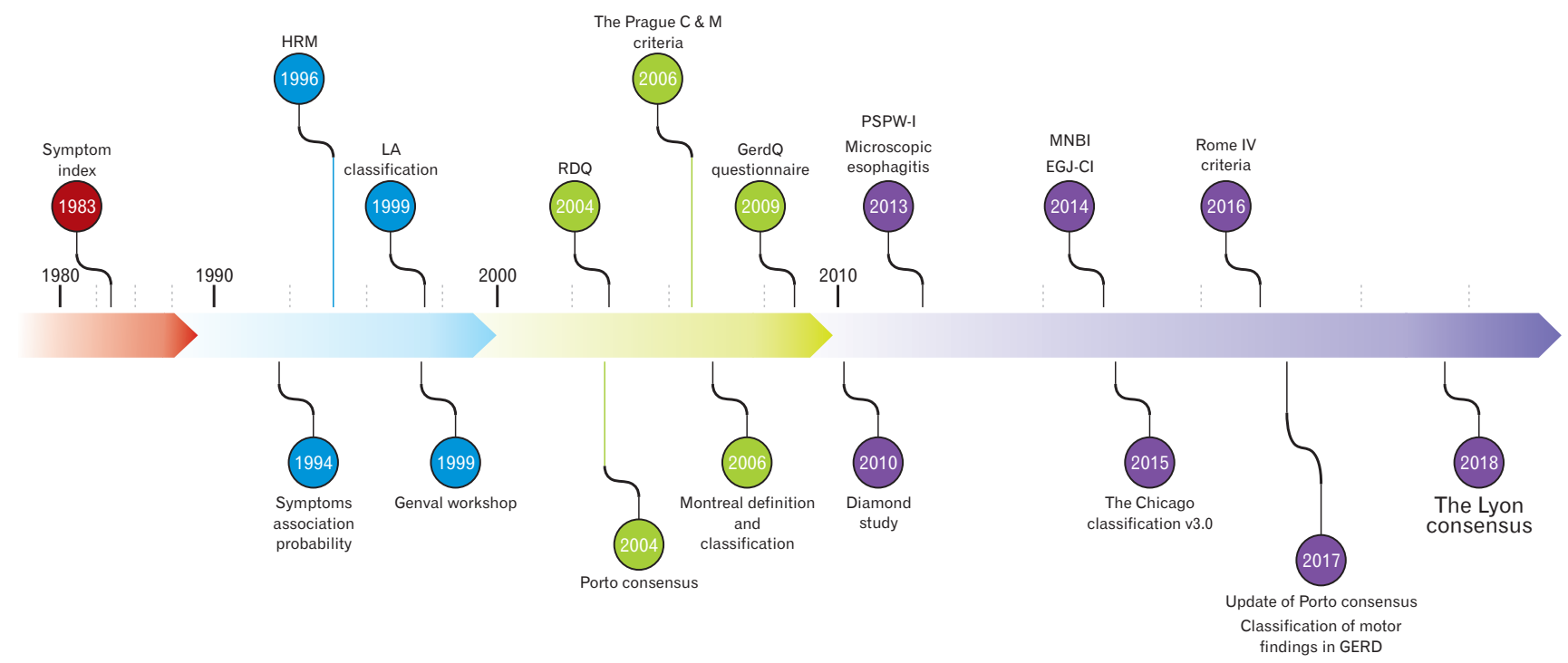

Figure 1. Time-line with the landmarks in the gastroesophageal reflux disease (GERD) assessment that have led to the Lyon Consensus. HRM, high-resolution manometry; LA, Los Angeles; RDQ, reflux disease questionnaire; GERDQ, gastroesophageal reflux disease questionnaire; PSPW-I, post-swallow reflux-induced peristaltic wave index; MNBI, mean nocturnal baseline impedance; EGJ-CI, esophagogastric junction contractile integral. 
loss, dysphagia, and digestive bleeding), when GERD is suspected, the PPI trial is a good empirical method to assess whether patient's symptoms are acid-related. Though, compared to GERD assessment made by esophagogastroduodenoscopy (EGD) and $\mathrm{pH}-$ metry, its sensitivity and specificity as diagnostic test reach $71 \%$ and $44 \%$, respectively. ${ }^{9,14,15}$ In addition, results are often equivocal. In fact, up to one-third of patients with esophagitis do not have symptom relief after PPI therapy and, on the contrary, more than one-third of patients with both normal endoscopy and $\mathrm{pH}$-metry, presents symptom improvement after PPIs. ${ }^{16,17}$ Moving into the field of EE symptoms, even though they are truly related to GER, the success of this trial declines significantly. ${ }^{18}$

\section{Upper Endoscopy, Esophageal Biopsies, and Objective Assessment of Extra-esophageal Syndromes}

EGD is one of the key tools for the diagnosis of GERD (Fig. 3). Even though it is not able to rule out GERD, EGD is able to detect signs of reflux with its complications or other diseases that could explain symptoms. It is usually performed in patients with red flags or in patients who do not respond to PPIs. In the Lyon Consensus, grade C or D esophagitis (according to Los Angeles classification), ${ }^{19}$ peptic strictures and Barrett's esophagus (longer than 1 $\mathrm{cm})$, have been classified as diagnostic for GERD. Unfortunately, GERD-related endoscopic findings were found in less than 30\% of patients with typical symptoms and most of them are even less severe, such as grade A and B esophagitis. Moreover, influence of PPI therapy should be taken into account. A study on 700 GERD patients has shown that $10 \%$ of them diagnosed as nonerosive reflux disease (NERD) were instead healed erosive reflux disease (ERD) patients. ${ }^{20}$ Indeed, the above mentioned pathognomonic features were found in less than $10 \%$ of patients with GERD symptoms. ${ }^{20}$ Furthermore, concerning grade A and B esophagitis, the former can be found in $10 \%$ of asymptomatic controls and, the latter has an important interobserver variability leading to misinterpreting its diagnostic role. ${ }^{8}$ All together, these considerations highlight how EGD can be really specific, but is burdened by low sensitivity in GERD diagnosis.

Histological sampling is an option that may help in the definition of a microscopic reflux-induced damage and in the differential diagnoses among GERD and other esophageal disorders. In the absence of macroscopic lesions, several histologic alterations due to chronic inflammation (eg, microscopic esophagitis, including dilated intercellular spaces) can be of help in the diagnosis of NERD. Interestingly, these changes represent a continuous spectrum of alterations progressively more frequent from functional esophageal disorders, through true NERD (ie, patients with normal endosco-

\section{Before Lyon Consensus}

- The role of questionnaires in diagnosing GERD in clinical practice or research setting is not distinguished.

- Proton pump inhibitor trial is suitable in clinical practice for its availability, as well as sensitivity in diagnosing GERD.

\section{After Lyon Consensus}

- It has been highlighted that the various questionnaires present similar sensitivity and specificity compared to accurate gastroenterological evaluation. They remain more useful in clinical trials than in clinical practice.

- Proton pump inhibitor trial is suitable in clinical practice for its good cost-effectiveness and user-friendliness. However, its low specificity may determine GERD overdiagnosis and proton pump inhibitor abuse.
Figure 2. Indications for questionnaire and proton pump inhibitor-trial use. GERD, gastroesophageal reflux disease.

\section{Before Lyon Consensus}

- The meaning of possible findings at endoscopy and histology was not reported.

- Bilitec was suggested for detection of duodenogastroesophageal reflux.

\section{After Lyon Consensus}

- LA grade C or D esophagitis, peptic strictures and Barrett's esophagus (Ionger than $1 \mathrm{~cm}$ ) are conclusive for GERD.

- Histology is important in differential diagnosis with EoE.

- Microscopic inflammation found in esophageal biopsies may be of additional value in characterizing patients with unclear diagnosis of GERD.

- Bilitec is no longer recommended for GERD diagnosis.

Figure 3. Interpretation of macroscopic and microscopic findings on endoscopy and role of Bilitec. GERD, gastroesophageal reflux disease; LA, Los Angeles classification; EoE, eosinophilic esophagitis. 
py, but abnormal esophageal acid exposure), to erosive esophagitis, adding important information for the differential diagnosis among patients without macroscopic lesions at EGD. ${ }^{21}$ Equally important, it is necessary to bear in mind that eosinophilic esophagitis (EoE) may mimic GERD in its clinical presentation and response to PPI therapy, although the symptoms of dysphagia or food impaction are very frequent. This disease can be ruled out with bioptic sampling from both the proximal and distal esophagus, although a diagnostic approach based on upper endoscopy with biopsies appears to be cost-effective in patients with refractory GERD without dysphagia only when the prevalence of $\mathrm{EoE}$ is $8 \%$ or greater. ${ }^{22,23}$

As to EE syndromes, an objective diagnosis is much more challenging. Reflux can cause irritation and mucosal inflammation of the upper aerodigestive tract, through direct contact or neuromediated reflex, and posterior laryngeal inflammation is generally considered as a sign of laryngopharyngeal reflux. However, different studies emphasized that these signs are poorly accurate because of a low interobserver concordance, variable intra-observer reliability, and their presence in a high percentage of healthy individuals. ${ }^{24,25}$ Other diagnostic options such as pepsin and bile assays and $\mathrm{pH}$ measurement in saliva and bronchoalveolar lavage fluid have shown disappointing results in this field and are not useful in clinical practice. ${ }^{9}$ Besides these methods, also other tests, such as Bilitec used to detect the presence of bilirubin in the refluxate, are no longer recommended in GERD assessment. ${ }^{8}$

\section{Ambulatory Reflux Monitoring: pH-Metry and Combined Impedance-pH Monitoring -}

Esophageal $\mathrm{pH}$-metry or combined $\mathrm{pH}$ and intraluminal impedance monitoring (MII-pH), are the most useful catheterbased techniques to assess GER (Fig. 4). In particular, MII-pH has the advantage to measure all physical (liquid, gas, or mixed) and chemical (acid, weakly acidic, and weakly alkaline) types of refluxes regardless of $\mathrm{pH}$ values. A different device measuring only $\mathrm{pH}$, such as Bravo (ie, a wireless system recording up to 96 hours) can increase the diagnostic yield in some patients by analyzing data obtained after the first 24 hours, but it is more expensive and not widely available. ${ }^{26}$

The main metrics of reflux monitoring are the acid exposure time (AET) and the number of reflux episodes (NRE). AET represents the most reproducible parameter and is predictive of good response to both medical and surgical therapy. ${ }^{26}$ It is a continuous metric, which proportionally correlates with the severity of reflux. The Lyon Consensus sets an AET cut-off value of $<4 \%$ as definitively normal and $>6 \%$ as clearly pathological. ${ }^{8}$ Among impedance metrics, NRE, including acid, weakly acidic, and weakly alkaline refluxes, is considered normal when less than 40 over 24 hours and abnormal when more than 80 over 24 hours. Intermediate values of both AET and NRE are inconclusive for GERD diagnosis, if considered alone. An abnormal NRE is a useful metric mostly in

\section{Before Lyon Consensus}

- Impedance-pH monitoring presents the higher sensibility and sensitivity for GERD diagnosis. $\mathrm{pH}$-metry is required to assess reflux acidity. Their role in management of GERD patients and clear cut-off values for their metrics are not yet defined.

- No mention about AET and NRE normal values.

- No mention about indications to perform reflux monitoring on or off therapy.

- Limited discussion about symptoms and reflux episodes association.

- Baseline impedance is abnormally low in erosive esophagitis and Barrett's esophagus.

\section{After Lyon Consensus}

- Esophageal pH-metry should be performed off-PPI, instead MII-pH can be performed both on or off $\mathrm{PPI}$ according to the following indications:

- MII-pH off-PPI in normal/LA grade A-B esophagitis, EE symptoms, evaluation before ARS, recurrent/persistent symptoms on-PPI, and/or after ARS.

- MII-pH on-PPI in patients with prior positive $\mathrm{pH}$ testing, LA grade C-D esophagitis,

Barrett's esophagus, and peptic strictures.

- AET < 4\% and NRE < 40/24 hr are normal, AET > 6\% and > 80/24 hr are abnormal. NRE can be of help in case of inconclusive AET values.

- SI and SAP provide information about reflux episodes and symptoms correlation; they predict success of both medical and surgical therapy.

- MNBI values correlates with degree of mucosal damage and response to both medical and surgical therapy, also in borderline AET patients. It is useful for GERD diagnosis.

- PSPW-index well differentiates erosive esophagitis from functional disorders and abnormal values are associated with response to therapies.
Figure 4. Indications and interpretation of ambulatory reflux monitoring. GERD, gastroesophageal reflux disease; AET, acid exposure time; NRE, number reflux episodes; PPI, proton pump inhibitor; MII-pH, combined $\mathrm{pH}$ and intraluminal impedance monitoring; LA, Los Angeles classification; EE, extra-esophageal; ARS, anti-reflux surgery; SI, symptoms index; SAP, symptom association probability; MNBI, mean nocturnal baseline impedance; PSPW, post-swallow reflux-induced peristaltic wave. 
borderline AET, even though is not enough on its own to predict response to medical therapy and to support anti-reflux surgery $(\mathrm{ARS}){ }^{26,27}$

\section{Symptom-Reflux Associations}

An important aspect emphasized by the Lyon Consensus about ambulatory reflux monitoring is the temporal association between reflux events and symptoms, recorded during the examination. Only symptoms preceded by a reflux episode in the previous $2 \mathrm{~min}-$ utes are considered associated to GER. Two scores, the symptoms index (SI) and the symptom association probability (SAP), are widely used. SI just represents the percentage of symptoms preceded by reflux events, while SAP is a measure of probability and is calculated by a complex statistical method. Both indexes have been shown to be predictive of the success of both medical and surgical therapy. ${ }^{28,29}$ These metrics are crucial in the differential diagnosis between reflux hypersensitivity $(\mathrm{RH})$ and functional heartburn $(\mathrm{FH}){ }^{20}$

\section{The Post-reflux Swallow-induced Peristaltic Wave Index and the Mean Nocturnal Baseline Impedance}

Beyond the above-mentioned parameters, other metrics have been developed in the last decades, including the post-reflux swallow-induced peristaltic wave (PSPW) index and the mean nocturnal baseline impedance (MNBI). They have shown significant pathophysiological importance, clinical reliability, and ability to increase the diagnostic yield of the impedance monitoring test. ${ }^{8}$

The baseline impedance (BI) is expression of the integrity of the esophageal mucosa. Conceptually, the impedance is inversely linked to increased dilation of intercellular spaces and tight junction dysfunction. In the Porto Consensus, abnormal low values of $\mathrm{BI}$ in patients with erosive esophagitis and Barrett's mucosa were already reported, but their diagnostic value was still unclear and not standardized. ${ }^{3}$ Nowadays, it is clear that a drop in BI values is proportional to the degree of mucosal damage, but we know that also refluxes and swallows are able to determine BI drops. ${ }^{30}$ Therefore, $\mathrm{BI}$ is now calculated during sleep, as a mean of 3 different nocturnal periods (MNBI). This interesting metric correlates with typical symptoms and is proportionally higher moving from erosive esophagitis to true NERD and $\mathrm{RH}$, and is normal in $\mathrm{FH}$ and healthy controls. ${ }^{31,32}$ A low MNBI $(<2292 \Omega)$ permits predicting a good response to anti-reflux therapy, better and independently from AET, and identifies patients with pathologic and borderline AET who respond to medical and surgical anti-reflux therapy. ${ }^{33,34}$ It also increases with the healing of esophagitis after successful PPI therapy. ${ }^{35,36}$ Similar correlation with esophageal inflammation has been demonstrated by a simple and specific through-the-scope device, made to assess impedance during endoscopy. ${ }^{37}$

The PSPW is an antegrade drop in impedance within 30 seconds of an impedance-detected reflux episode. The proportion of refluxes followed by a PSPW is called PSPW index and reflects the integrity of primary peristalsis and chemical clearance stimulated by reflux episodes. This metric presents excellent ability to differentiate erosive esophagitis and pathological AET from $\mathrm{FH}$ and healthy controls, with a sensitivity and specificity of about $100 \%$ and $92 \%$, respectively. ${ }^{38}$ It also correlates with muscle contraction reserve assessed by multiple rapid swallows (MRS). ${ }^{39}$ At off-PPI impedance-pH tracing analysis, PSPW index is an independent factor associated with PPI success with abnormal values in $92 \%$ of PPI-responsive patients. ${ }^{40}$ Evaluating on-PPI MII-pH tracings, PSPW values were significantly and progressively lower from patients with $\mathrm{FH}$ to NERD, healed reflux esophagitis and refractory esophagitis. ${ }^{41}$ Abnormal values, that describe the underlying mechanism, are associated with a satisfactory response to acid-suppressive therapy, also in patients with EE syndromes. ${ }^{42}$ However, it should be also noted that PPI-refractory esophagitis presents lower values of PSPW index, that explains the pathophysiological reason responsible for the lack of response. ${ }^{41}$

\section{On-/Off-Proton Pump Inhibitor Therapy pH- Impedance Monitoring Assessment}

Another important aspect stressed in the Lyon Consensus is the indication to perform MII-pH with or without anti-secretive therapy. As it can be easily guessed, PPI therapy can significantly modify the results of MII-pH tracings as well as those of $\mathrm{pH}$ metry monitoring, and therefore PPIs should be always discontinued before these examinations. ${ }^{8}$ Using MII-pH, reflux should be assessed off-PPI in most patients, like those who have an unproven GERD (without peptic lesions or with the lower grades of erosive esophagitis at EGD), EE symptoms, under evaluation for ARS, or with recurrent/persistent symptoms on PPIs and/or after surgery. Tests should be performed on PPI in proven GERD, that is in patients with prior positive $\mathrm{pH}$-testing, esophagitis with Los Angeles grades $\mathrm{C}$ and $\mathrm{D}$, Barrett's esophagus, and peptic strictures. ${ }^{8,26}$ The reason for this subdivision is that in the former subset of patients the aim of reflux monitoring is to understand whether a pathological reflux is present or not. On the contrary, in those with already known GERD, the aim is to understand why symptoms or lesions are refractory to anti-secretive therapy. It is important to remember that on-PPI MII-pH is required in order to measure weakly acidic 
refluxes, which represent the majority with anti-secretive therapy. ${ }^{8,26}$ Even though MII-pH permits to analyze the symptom-reflux association also on-PPI, the evaluation off-PPI increases its sensitivity because of a higher number of symptoms reported.

\section{High-resolution Esophageal Manometry}

Usually HRM is performed at the same time as reflux monitoring probe placement in order to identify anatomical markers of esophagogastric junction (EGJ). Evaluation of normal esophageal peristalsis and exclusion of esophageal motility disorders, are also mandatory before ARS. In the last decade, several motor abnormalities were found in GERD patients and were recently classified by Gyawali et al. ${ }^{43} \mathrm{HRM}$ is able to study the EGJ in terms of a barrier against gastroesophageal reflux, better than $\mathrm{EGD} .{ }^{43} \mathrm{EGJ}$ is a complex dynamic structure, in which the lower esophageal sphincter (LES) and crural diaphragm (CD) interact to maintain this valve functioning during acts of breathing, swallowing, and changes of body position. Even though some degree of EGJ incompetence is physiological, as during transient lower esophageal sphincter relaxation (TLESR), it creates an open gate that allows the back migration of gastric content into the esophagus. ${ }^{8,43}$

Evaluation of EGJ consists of its anatomical assessment with identification or exclusion of hiatal hernia and evaluation of EGJ contractile vigor (Fig. 5). Indeed, EGJ has been subdivided into 3 types, based on the relationship between the LES and $\mathrm{CD}$, that can be superimposed (type I), slightly ( $<3 \mathrm{~cm}$, type II), or markedly ( $>$ $3 \mathrm{~cm}$, type III) separated. Increasing the separation between LES and $\mathrm{CD}$ can cause a gradual and significant increase in reflux, and mostly the type III is related to reflux severity and greater positive symptom-reflux association, due to the loss of its barrier role. ${ }^{44}$ As to motor function, EGJ contractile integral (EGJ-CI) is a promising parameter, that consists of the distal contractile integral (DCI) applied to LES and CD, calculated among 3 respiratory cycles. Low values correlate with both ERD and abnormal ambulatory reflux monitoring scores. ${ }^{45}$ Wide differences among mean values in different studies highlight difficulties in its calculation. The Lyon Consensus recommends to standardize methodology among groups, with the exclusion of the $\mathrm{CD}$ component of EGJ in instances of type III EGJ morphology and to calculate EGJ-CI above gastric baseline pressure. ${ }^{8}$

Another important aspect related to EGJ is the assessment of TLESR defined as LES relaxation occurring in absence of swallowing, lasting more than 10 seconds, and associated with $\mathrm{CD}$ inhibition. ${ }^{46}$ This is a physiologic event that allows passage of air from stomach to esophagus as a belch. In GERD patients TLESRs are prolonged with profound LES relaxation, that is accompanied by reflux of gastric content. ${ }^{43}$ Although TLESRs are the most common EGJ event in patients with GERD, they are not usually evaluated on HRM. ${ }^{27}$ Besides, HRM performed during postprandial periods or test meals, even if not yet standardized, could be of interest in the future to measure the number of TLESRs and other disorders (eg, rumination syndromes). ${ }^{8}$

Esophageal peristalsis is also involved in GERD pathophysiology. Usually manometric tracings of GERD patients are normal, but among the abnormal ones, hypomotility disorders are the most frequent findings. ${ }^{43}$ The burden of reflux symptoms and AET values present a descending gradient from absent, ineffective, and fragmented peristalsis. ${ }^{43}$ AET values increase proportionally with defects of esophageal peristalsis like weak, failed, and absent peristalsis traces. ${ }^{47,48}$ The likelihood of an abnormal peristaltic tracing is higher in erosive esophagitis and Barrett's esophagus compared to NERD, demonstrating the involvement of esophageal body in reflux clearance. ${ }^{43}$ However, beyond the relationship between esophageal motor findings and GERD, pathophysiological connections between them are still unclear. Motility abnormalities do not necessarily improve after erosive esophagitis healing, suggesting a possible primary motor disorder as responsible for GERD development, or an irreversible motor injury due to peptic action. It has been also documented that dysmotility improves after ARS, show-

\section{Before Lyon Consensus}

\section{- No discussion about esophageal motor function in GERD.}

\section{After Lyon Consensus}

A systematic and standardized assessment with esophageal high-resolution manometry is suggested: EGJ anatomy (type I-III) and vigor (EGJ-Cl); presence of transient lower esophageal sphincter relaxations; characteristics of esophageal peristalsis (absent, ineffective or fragmented peristalsis); contractile reserve (distal contractile integral ratio between wet swallows and multiple rapid swallows); rapid drinking challenge to assess subclinical esophageal outflow obstruction.

- EGJ barrier dysfunction, ineffective clearance of esophageal body and low contraction reserve are related with the burden of GERD symptoms and lesions.
Figure 5. Characterization and interpretation of motor findings in gastroesophageal reflux disease (GERD) patients. EGJ, esophagogastric junction; $\mathrm{CI}$, contractile integral. 
ing how some patients can restore their esophageal motor function. In contrast, NERD, RH and FH may have increased vigor of distal esophageal contraction compared to disorders with pathological AET. $^{43}$

Contraction reserve is the physiological phenomenon characterized by the increase of esophageal body contraction, following provocative maneuvers. It is due to the forced inhibition of the contraction of the smooth esophageal muscle and relaxation of the LES during the consecutive wet swallows, followed by a vigorous peristaltic wave. Provocative tests are usually performed in the latter part of a manometric evaluation. Rapid drinking challenge (RDC) and MRS are usually performed and standardized among centers, instead test meals are less frequently applied. After multiple wet swallows in rapid succession, as in MRS, the contraction wave should present greater DCI than normal swallows. Lyon Consensus suggests a ratio between post-MRS and pre-MRS DCI (peristaltic augmentation ratio) to assess this reserve, that is adequate when it is greater than $1 .^{49}$ Erosive esophagitis patients frequently present an ineffective contraction reserve compared to NERD and healthy controls, and it can represent the reason why they have an increased exposure to gastric refluxate. ${ }^{50}$ The peristaltic augmentation ratio is also related with higher AET in NERD and seems to predict the development of ineffective motility after ARS. ${ }^{39}$ Nowadays the RDC finds less application in GERD assessment, being more useful in achalasia and EGJ outflow obstruction differential diagnosis, thanks to its ability to highlight resistance to bolus passage across the EGJ. However, an effective post-RDC peristalsis was shown in only $30 \%$ of patients with ERD, compared to $80 \%$ of healthy controls. ${ }^{8}$

Bearing in mind the above mentioned manometric metrics, the Lyon Consensus recommends a step-by-step approach, which involves, first of all, the EGJ assessment (morphology and EGJ-CI), then the study of body peristalsis, and lastly the contraction reserve (Fig. 5). The Chicago classification version 3.0 should be taken as reference for HRM interpretation. ${ }^{51}$

\section{Discussion and Conclusion}

The great importance of the Lyon Consensus has been the focus on the available diagnostic tools for GERD assessment (Fig. 6). Compared to statements previously proposed by the Porto Consensus, ${ }^{3}$ the indications now provided have been updated in order to standardize GERD management, giving clear and useful indications for the choice and interpretation of the various tests. Findings as severe esophagitis, Barrett's esophagus, peptic strictures, and AET $>6 \%$ allows us to diagnose GERD definitely. On the contrary, even if EGD is not able to rule out GERD, when accompanied by normal $\mathrm{pH}$-metry, it provides clear evidence against a reflux-mediated disease. According to the Lyon Consensus the approach is standardized instead of being based on the result of a single metric, and therefore the more accurate diagnosis of GERD is favored. Furthermore, the old concept of GERD as a single clini-

\begin{tabular}{|c|c|c|c|c|}
\hline Diagnosis & Upper endoscopy & Reflux monitoring & Response to PPI & HRM \\
\hline GERD & $\begin{array}{c}\text { LA grade C \& D esophagitis, } \\
\text { peptic strictures, } \\
\text { Barrett's esophagus }\end{array}$ & AET $>6 \%$ & High & / \\
\hline True NERD & Normal & $\mathrm{AET}>6 \%$ & High-intermediate & l \\
\hline Reflux hypersensitivity & Normal & $\begin{array}{c}\text { AET }<4 \% ; \text { SI > } 50 \% \\
\text { SAP > } 95 \%\end{array}$ & Low-intermediate & / \\
\hline Functional heartburn & Normal & $\begin{array}{c}\text { AET }<4 \% ; \text { SI }<50 \% \\
\text { SAP }<95 \%\end{array}$ & Low & I \\
\hline Additional evaluations & Upper endoscopy & Reflux monitoring & Response to PPI & HRM \\
\hline $\begin{array}{l}\text { Results suggestive, } \\
\text { but inconclusive } \\
\text { for reflux }\end{array}$ & $\begin{array}{c}\text { LA grade A \& B esophagitis, } \\
\text { microscopic inflammation } \\
\text { at biopsies }\end{array}$ & $\begin{array}{c}\text { AET }=4-6 \% \\
\text { Number of refluxes }>80 / 24 \mathrm{hr} \\
\text { Low MNBI; Low PSPW index }\end{array}$ & / & $\begin{array}{l}\text { EGJ type III, } \\
\text { low EGJ-Cl; } \\
\text { Esophageal } \\
\text { hypomotility }\end{array}$ \\
\hline Results against reflux & / & $\begin{array}{c}\text { AET }<4 \% \\
\text { Number of refluxes }<40 / 24 \mathrm{hr}\end{array}$ & I & $\begin{array}{l}\text { Achalasia or EGJ- } \\
\text { outflow obstruction }\end{array}$ \\
\hline
\end{tabular}

Figure 6. Esophageal test results in gastroesophageal reflux disease (GERD) assessment. PPI, proton pump inhibitor; HRM, high-resolution manometry; NERD, nonerosive reflux disease; LA, Los Angeles classification; AET, acid exposure time; SI, symptom index; SAP, symptom association probability; MNBI, mean nocturnal baseline impedance; PSPW, post-swallow reflux-induced peristaltic wave; EGJ, esophagogastric junction; CI, contractile integral. 
cal, pathophysiological entity has been overcome, because it permits exploration of a spectrum of different diseases with similar presentations, which have separate pathogenesis and therapeutic implications.

Specialists usually have to take into consideration this guided algorithm in patients that do not respond to PPI therapy. After interventions made to optimize the anti-secretory therapy, the first investigation that patients should undergo is, if not already performed, an EGD. Early upper endoscopy is indicated only in presence of red flags and can identify either GERD lesions or other findings that can explain symptoms, such as peptic ulcer, gastric adenocarcinoma or other types of esophagitis (EoE, drug-induced, and infectious). However, most of these patients present normal endoscopy. No clear indication of esophageal biopsies during EGD are reported in the Lyon Consensus, unless in the absence of macroscopic findings or in the clinical suspicion of EoE.

The next step is the correct sub-categorization of those patients who, although presenting symptoms suggestive for GERD, have a normal EGD. These patients can be true NERD or patients with functional disorders of the esophagus (about 60\%). True NERD is characterized by pathological $\mathrm{pH}$-metry/MII-pH tracings and, for this reason, the relationship between GER and clinical symptoms is highly probable. On the contrary, patients with normal records might have a functional disorder of the esophagus or RH. The latter diagnosis is characterized by normal AET, but positive association between symptoms and reflux episodes, in contrast with $\mathrm{FH}$, that not only presents with normal MII-pH, but has also negative SI and SAP. Two crucial elements to integrate the standard MII$\mathrm{pH}$ analysis are the PSPW index and the MNBI, that independently and optimally correlate with a reflux-mediated pathogenesis of symptoms and could clarify pathophysiological doubts. Unfortunately, until now, no pathognomonic impedance patterns for PPI non-responders have been found.

However, it is not always so easy to achieve the right diagnosis. There are some grey areas, such as low-grade esophagitis and AET between 4-6\%, that need further clues to be clarified. In this context, a microscopic inflammation at bioptic esophageal samples, a pathological NRE and manometric features of EGJ dysfunction and/or hypotensive esophageal motility, can provide diagnostic support. Nevertheless, it remains challenging to decide the best therapeutic approach, including ARS, in patients with AET between 4 and 6. Further studies are required in this particular setting.

The combined evaluation of the level of refluxate exposure, mechanism of reflux, efficacy of clearance, underlying anatomy of the EGJ and psychometrics defining symptomatic presentations, can guide through this intricate labyrinth in order to assess, in every patient, his/her own specific phenotype. A correct definition is fundamental for a tailored therapy, considering that available options range from behavioural therapy to ARS, through a wide variety of drugs that are not only PPIs.

\section{Five-year Prospective}

GERD management strategies should focus on defining individual patient phenotype taking into account their characteristics. As the GERD diagnostic paradigm evolves, also a precise approach tailored to the single patient becomes possible, going beyond a dichotomic definition based on the presence or absence of GERD. Firstly, we need to continue improving our knowledge about GERD pathophysiology. So far, the majority of data available in this field comes from monocentric studies analyzing patients non-responsive to PPI therapy or candidate for ARS. We should encourage the development of prospective, multicentric and standardized studies, matched with large control groups, in order to define the relevant variations of available metrics among the various types of patients and to better understand the underlying pathophysiology.

In parallel with the wider popularity of ambulatory reflux monitoring, guidelines and protocols as well as software analysis methods should improve at the same time. While the assessment of AET is automatic and reliable, the manual review of the tracing is still required for NRE in consideration of problems related to software overestimation. Moreover, at present, PSPW and MNBI need to be calculated manually also. If we consider the global calculation of all the metrics available today, the analysis of one $\mathrm{pH}-\mathrm{MII}$ tracing takes about 20-30 minutes, a workload that can hardly be done by a specialist in relation to labor-intensity, considering the intense activity of digestive pathophysiology units. Therefore, integration of these metrics into the automatic standard ambulatory reflux monitoring assessment is needed.

A great progress has been made also in HRM, although the new classification of motor findings in GERD was missed in the Chicago classification version 3.0. EGJ-CI and provocative tests, as MRS and RDC, both help to manage GERD, but further forward steps are expected. The test meal is still a poor standardized and investigated metric. ${ }^{52}$ Administration of a meal during the HRM procedure can be useful to highlight disorders hidden to a standard analysis with wet swallows. The combination of impedance with HRM allows a comprehensive evaluation of the esophageal function, including bolus transit and clearance. Even though a correlation between esophageal motor abnormalities and 
bolus transit defects in different subgroups of GERD patients has been already demonstrated, this methodology is not widely used, due to its complexity and costs. ${ }^{53}$ Impedance with HRM after a test meal is helpful in the differential diagnosis with conditions that can mimic GERD, such as rumination syndrome and supragastric belching. ${ }^{54}$ This step is important, considering that these disorders can benefit more from behavioral interventions than from medical therapy or ARS. In a retrospective study with a non-standardized test meal, rumination events and supragastric belching were reported in $20 \%$ and $42 \%$ of cases, respectively, of GERD patients, who were diagnosed as PPI non-responders. ${ }^{55}$ Indeed, we suggest that future guidelines and recommendations from national societies propose provocation test with standardized meal for the evaluation of GERD patients.

The 3-dimensional HRM analysis of the EGJ and the functional lumen imaging probe (FLIP), instead, probably need further studies to understand their real usefulness. FLIP has shown increased EGJ compliance in GERD patients compared with healthy controls, that explains the increased volumes of reflux in the former ones. ${ }^{56}$ However, controversial results about its helpfulness have arrived from studies that applied this method in the setting of ARS. ${ }^{57,58}$

With a greater familiarity with available metrics, we need to better understand what is behind esophageal functional disorders. While it is well known that Barrett's esophagus may be associated with esophageal hyposensitivity, correlation between hypersensitivity, hypervigilance, and GERD symptoms remains unclear and not completely understood. ${ }^{59}$

Lastly, greater attention should be taken in the assessment of $\mathrm{EE}$ syndromes. The new insights achieved into pharyngoesophageal motor function and upper esophageal sphincter dysfunction, are far from a complete comprehension of pathophysiological alterations underlying EE syndromes. As mis- and over-diagnosis lead to low efficacy of anti-reflux therapies, a more rigorous and standardized approach to these patients will improve management and outcomes.

\section{Financial support: None.}

\section{Conflicts of interest: None.}

Author contributions: Matteo Ghisa, Brigida Barberio, Vincenzo Savarino, Elisa Marabotto, Mentore Ribolsi, Giorgia Bodini, Fabiana Zingone, Marzio Frazzoni, and Edoardo Savarino: data collection, writing of the manuscript, and approving final version; and Vincenzo Savarino: guarantor of this article.

\section{References}

1. Vakil N, van Zanten SV, Kahrilas P, Dent J, Jones R. The Montreal definition and classification of gastroesophageal reflux disease: a global evidence-based consensus. Am J Gastroenterol 2006;101:1900-1920.

2. Ronkainen J, Agréus L. Epidemiology of reflux symptoms and GORD. Best Pract Res Clin Gastroenterol 2013;27:325-337.

3. Sifrim D, Castell D, Dent J, Kahrilas PJ. Gastro-oesophageal reflux monitoring: review and consensus report on detection and definitions of acid, non-acid, and gas reflux. Gut 2004;53:1024-1031.

4. Dent J, Brun J, Fendrick A, et al. An evidence-based appraisal of reflux disease management--the Genval workshop report. Gut 1999;44(suppl 2):S1-S16.

5. Katz PO, Gerson LB, Vela MF. Guidelines for the diagnosis and management of gastroesophageal reflux disease. Am J Gastroenterol 2013;108:308-328.

6. Savarino V, Marabotto E, Zentilin P, et al. Proton pump inhibitors: use and misuse in the clinical setting. Expert Rev Clin Pharmacol 2018;11:1123-1134.

7. Savarino V, Dulbecco P, de Bortoli N, Otonello A, Savarino E. The appropriate use of proton pump inhibitors (PPIs): need for a reappraisal. Eur J Intern Med 2017;37:19-24.

8. Gyawali CP, Kahrilas PJ, Savarino E, et al. Modern diagnosis of GERD: the Lyon Consensus. Gut 2018;67:1351-1362.

9. Dent J, Vakil N, Jones R, et al. Accuracy of the diagnosis of GORD by questionnaire, physicians and a trial of proton pump inhibitor treatment: the diamond study. Gut 2010;59:714-721.

10. Ghisa M, Della Coletta MD, Barbuscio I, et al. Updates in the field of non-esophageal gastroesophageal reflux disorder. Expert Rev Gastroenterol Hepatol 2019;13:827-838.

11. Ghisa M, Marinelli C, Savarino V, Savarino E. Idiopathic pulmonary fibrosis and GERD: links and risks. Ther Clin Risk Manag 2019;15:1081-1093.

12. Bolier EA, Kessing BF, Smout AJ, Bredenoord AJ. Systematic review: questionnaires for assessment of gastroesophageal reflux disease. Dis Esophagus 2015;28:105-120.

13. Jones R, Junghard O, Dent J, et al. Development of the GerdQ, a tool for the diagnosis and management of gastro-oesophageal reflux disease in primary care. Aliment Pharmacol Ther 2009;30:1030-1038.

14. Savarino E, Marabotto E, Zentilin P, et al. A safety review of proton pump inhibitors to treat acid-related digestive diseases. Expert Opin Drug Saf 2018;17:785-794.

15. Sifrim D, Zerbib F. Diagnosis and management of patients with reflux symptoms refractory to proton pump inhibitors. Gut 2012;61:13401354.

16. Bytzer P, Jones R, Vakil N, et al. Limited ability of the proton-pump inhibitor test to identify patients with gastroesophageal reflux disease. Clin Gastroenterol Hepatol 2012;10:1360-1366.

17. Numans ME, Lau J, de Wit NJ, Bonis PA. Short-term treatment with proton-pump inhibitors as a test for gastroesophageal reflux disease: a meta-analysis of diagnostic test characteristics. Ann Intern Med 
2004;140:518-527.

18. Gyawali CP, Fass R. Management of gastroesophageal reflux disease. Gastroenterology 2018;154:302-318.

19. Lundell LR, Dent J, Bennett JR, et al. Endoscopic assessment of oesophagitis: clinical and functional correlates and further validation of the Los Angeles classification. Gut 1999;45:172-180.

20. Gaddam S, Wani S, Ahmed H, et al. The impact of pre-endoscopy proton pump inhibitor use on the classification of non-erosive reflux disease and erosive oesophagitis. Aliment Pharmacol Ther 2010;32:1266-1274.

21. Savarino E, Zentilin P, Savarino V. NERD: an umbrella term including heterogeneous subpopulations. Nat Rev Gastroenterol Hepatol 2013;10:371-380.

22. Savarino E, Zentilin P, Mastracci L, et al. Microscopic esophagitis distinguishes patients with non-erosive reflux disease from those with functional heartburn. J Gastroenterol 2013;48:473-482.

23. Miller SM, Goldstein JL, Gerson LB. Cost-effectiveness model of endoscopic biopsy for eosinophilic esophagitis in patients with refractory GERD. Am J Gastroenterol. 2011;106:1439-1445.

24. Milstein CF, Charbel S, Hicks DM, Abelson TI, Richter JE, Vaezi MF. Prevalence of laryngeal irritation signs associated with reflux in asymptomatic volunteers: impact of endoscopic technique (rigid vs. flexible laryngoscope). Laryngoscope 2005;115:2256-2261.

25. Branski RC, Bhattacharyya N, Shapiro J. The reliability of the assessment of endoscopic laryngeal findings associated with laryngopharyngeal reflux disease. Laryngoscope 2002;112:1019-1024.

26. Roman S, Gyawali CP, Savarino E, et al. Ambulatory reflux monitoring for diagnosis of gastro-esophageal reflux disease: update of the Porto consensus and recommendations from an international consensus group. Neurogastroenterol Motil 2017;29:1-15.

27. Savarino E, Bredenoord AJ, Fox M, et al. Expert consensus document: advances in the physiological assessment and diagnosis of GERD. Nat Rev Gastroenterol Hepatol 2017;14:665-676.

28. Patel A, Sayuk GS, Gyawali CP. Parameters on esophageal pH-impedance monitoring that predict outcomes of patients with gastroesophageal reflux disease. Clin Gastroenterol Hepatol 2015;13:884-891.

29. Desjardin M, Luc G, Collet D, Zerbib F. 24-hour pH-impedance monitoring on therapy to select patients with refractory reflux symptoms for antireflux surgery. a single center retrospective study. Neurogastroenterol Motil 2016;28:146-152.

30. Farré R, Blondeau K, Clement D, et al. Evaluation of oesophageal mucosa integrity by the intraluminal impedance technique. Gut 2011;60:885892.

31. de Bortoli N, Martinucci I, Savarino E, et al. Association between baseline impedance values and response proton pump inhibitors in patients with heartburn. Clin Gastroenterol Hepatol 2015;13:1082-1088, e1.

32. Frazzoni M, Savarino E, de Bortoli N, et al. Analyses of the post-reflux swallow-induced peristaltic wave index and nocturnal baseline impedance parameters increase the diagnostic yield of impedance-pH monitoring of patients with reflux disease. Clin Gastroenterol Hepatol 2016;14:40-46.

33. Patel A, Wang D, Sainani N, Sayuk GS, Gyawali CP. Distal mean nocturnal baseline impedance on $\mathrm{pH}$-impedance monitoring predicts reflux burden and symptomatic outcome in gastro-oesophageal reflux disease.
Aliment Pharmacol Ther 2016;44:890-898.

34. Rengarajan A, Savarino E, Della Coletta M, Ghisa M, Patel A, Gyawali CP. Mean nocturnal baseline impedance correlates with symptom outcome when acid exposure time is inconclusive on esophageal reflux monitoring. Clin Gastroenterol Hepatol 2019;18:589-595.

35. van Rhijn BD, Weijenborg PW, Verheij J, et al. Proton pump inhibitors partially restore mucosal integrity in patients with proton pump inhibitorresponsive esophageal eosinophilia but not eosinophilic esophagitis. Clin Gastroenterol Hepatol 2014;12:1815-1823, e2.

36. Frazzoni M, Penagini R, Frazzoni L, et al. Role of reflux in the pathogenesis of eosinophilic esophagitis: comprehensive appraisal with off- and on PPI impedance-pH monitoring. Am J Gastroenterol 2019;114:16061613.

37. Katzka DA, Ravi K, Geno DM, et al. Endoscopic mucosal impedance measurements correlate with eosinophilia and dilation of intercellular spaces in patients with eosinophilic esophagitis. Clin Gastroenterol Hepatol 2015;13:1242-1248, e1.

38. Frazzoni M, Savarino E, de Bortoli N, et al. Analyses of the post-reflux swallow-induced peristaltic wave index and nocturnal baseline impedance parameters increase the diagnostic yield of impedance-pH monitoring of patients with reflux disease. Clin Gastroenterol Hepatol 2016;14:40-46.

39. Martinucci I, Savarino EV, Pandolfino JE, et al. Vigor of peristalsis during multiple rapid swallows is inversely correlated with acid exposure time in patients with NERD. Neurogastroenterol Motil 2016;28:243-250.

40. Frazzoni L, Frazzoni M, de Bortoli N, et al. Postreflux swallow-induced peristaltic wave index and nocturnal baseline impedance can link PPIresponsive heartburn to reflux better than acid exposure time. Neurogastroenterol Motil 2017;29:e13116.

41. Frazzoni M, de Bortoli N, Frazzoni L, et al. The added diagnostic value of postreflux swallow-induced peristaltic wave index and nocturnal baseline impedance in refractory reflux disease studied with on-therapy impedance-pH monitoring. Neurogastroenterol Motil 2017;29:e12947.

42. Ribolsi M, Guarino MPL, Tullio A, Cicala M. Post-reflux swallowinduced peristaltic wave index and mean nocturnal baseline impedance predict PPI response in GERD patients with extra esophageal symptoms. Dig Liver Dis 2020;52:173-177.

43. Gyawali CP, Roman S, Bredenoord AJ, et al. Classification of esophageal motor findings in gastro-esophageal reflux disease: Conclusions from an international consensus group. Neurogastroenterol Motil 2017;29:e13104.

44. Tolone S, de Cassan C, de Bortoli N, et al. Esophagogastric junction morphology is associated with a positive impedance-pH monitoring in patients with GERD. Neurogastroenterol Motil 2015;27:1175-1182.

45. Tolone S, De Bortoli N, Marabotto E, et al. Esophagogastric junction contractility for clinical assessment in patients with GERD: a real added value? Neurogastroenterol Motil 2015;27:1423-1431.

46. Roman S, Holloway R, Keller J, et al. Validation of criteria for the definition of transient lower esophageal sphincter relaxations using highresolution manometry. Neurogastroenterol Motil 2017;29:e12920.

47. Rengarajan A, Bolkhir A, Gor P, Wang D, Munigala S, Gyawali CP. Esophagogastric junction and esophageal body contraction metrics on high-resolution manometry predict esophageal acid burden. Neurogas- 
troenterol Motil 2018;30:e13267.

48. Reddy CA, Patel A, Gyawali CP. Impact of symptom burden and healthrelated quality of life (HRQOL) on esophageal motor diagnoses. Neurogastroenterol Motil 2017;29:e12970.

49. Carlson DA, Crowell MD, Kimmel JN, et al. Loss of peristaltic reserve, determined by multiple rapid swallows, is the most frequent esophageal motility abnormality in patients with systemic sclerosis. Clin Gastroenterol Hepatol 2016;14:1502-1506.

50. Daum C, Sweis R, Kaufman E, et al. Failure to respond to physiologic challenge characterizes esophageal motility in erosive gastro-esophageal reflux disease. Neurogastroenterol Motil 2011;23:517-e200.

51. Kahrilas PJ, Bredenoord AJ, Fox M, et al. The Chicago classification of esophageal motility disorders, v3.0. Neurogastroenterol Motil 2015;27:160-174.

52. Ang D, Misselwitz B, Hollenstein M, et al. Diagnostic yield of highresolution manometry with a solid test meal for clinically relevant, symptomatic oesophageal motility disorders: serial diagnostic study. Lancet Gastroenterol Hepatol 2017;2:654-661.

53. Savarino E, Gemignani L, Pohl D, et al. Oesophageal motility and bolus transit abnormalities increase in parallel with the severity of gastrooesophageal reflux disease. Aliment Pharmacol Ther 2011;34:476-486.

54. Bredenoord AJ, Tutuian R, Smout AJ, Castell DO, et al. Technol- ogy review: esophageal impedance monitoring. Am J Gastroenterol 2007;102:187-194.

55. Yadlapati R, Tye M, Roman S, Kahrilas PJ, Ritter K, Pandolfino JE. Postprandial high-resolution impedance manometry identifies mechanisms of nonresponse to proton pump inhibitors. Clin Gastroenterol Hepatol 2018;16:211-218, e1.

56. Kwiatek MA, Pandolfino JE, Hirano I, Kahrilas PJ. Esophagogastric junction distensibility assessed with an endoscopic functional luminal imaging probe (EndoFLIP). Gastrointest Endosc 2010;72:272-278.

57. Hirano I, Pandolfino JE, Boeckxstaens GE. Functional lumen imaging probe for the management of esophageal disorders: expert review from the clinical practice updates committee of the AGA institute. Clin Gastroenterol Hepatol 2017;15:325-334.

58. Smeets FG, Keszthelyi D, Bouvy ND, Masclee AA, Conchillo JM. Does measurement of esophagogastric junction distensibility by EndoFLIP predict therapyresponsiveness to endoluminal fundoplication in patients with gastroesophageal reflux disease? J Neurogastroenterol Motil 2015;21:255-264

59. Carlson DA, Gyawali CP, Roman S, et al. Esophageal hypervigilance and visceral anxiety are contributors to symptom severity among patients evaluated with high-resolution esophageal manometry. Am J Gastroenterol 2020;115:367-375. 